\title{
On the Thermodynamics of Urban Environments
}

\section{The Thermal Potential of the Cities}

\author{
Nilo Serpa \\ Faculdade ICESP de Brasília, Brasil.
}

Received:_15 May 2018__ / Accepted:__04 September 2018___ Published:_20 October 2018

\begin{abstract}
Present article proposes the application of the concept of entropy in the study of the environmental impacts generated by a city. It is taken a strictly thermodynamic approach of cities as heat sinks that generate entropy. The formalism of caloric field theory is adopted to establish the relationship between entropy caused by thermal exchanges and the physical environmental parameters considered. The notion of evolutional global time is introduced in order to treat entropy more objectively in the context of thermodynamics. A table with the rates of change of entropy in some cities is generated.
\end{abstract}

Key words: Entropy, thermodynamics, irreversible processes, caloric field theory.

Resumo: O presente artigo propõe a aplicação do conceito de entropia no estudo dos impactos ambientais gerados por uma cidade. É feita uma abordagem estritamente termodinâmica das cidades como dissipadores térmicos geradores de entropia. O formalismo da teoria dos campos calóricos é adotado para estabelecer a relação entre a entropia produzida pelas trocas térmicas e os parâmetros físicos ambientais considerados. O conceito de tempo global evolutivo é introduzido por modo de tratar a entropia de maneira mais objetiva no contexto da termodinâmica. É gerada uma tabela com as taxas de variação da entropia em algumas cidades.

Palavras-chave: Entropia, termodinâmica, processos irreversíveis, teoria de campo calórico.

\section{Introduction}

The concept of entropy is one of the least understood and at the same time one of the most evoked in sciences, pseudo-sciences and semi-sciences. Bunge, in his Dictionary of Philosophy, observes that: "Há dois conceitos técnicos de entropia

Corresponding Author: Nilo Silvio Costa Serpa, ICESP, nilo.serpa@icesp.edu.br. diferentes e não relacionados, a saber: o físico e o informacional. Nenhum deles é relevante para a filosofia, embora a palavra 'entropia' seja uma favorita entre os filósofos pops" [2]. Also, Bunge highlights a common wrong idea: “[...] the interpretation of entropy as a measure of our ignorance is invalid, for it involves the erroneous identification of

\footnotetext{
1 There are two different and unrelated technical concepts of entropy, namely: the physical and the informational. None of them is relevant to philosophy, although the word 'entropy' is a favorite among pop philosophers. (author's free translation).
} 
statistical mechanics with epistemology" [1], and he continues saying that it is abusive to "[...] reduce entropy increase to loss of human information about the system, for this deprives statistical mechanics and thermodynamics of objectivity" [1]. Most of these claims are correct except for one. I would agree with Bunge on the lack of value of the concept of entropy for philosophy if he had not repeated the misconception of negentropy [1]. The fact is that there is no "negative entropy", but rather "entropy deceleration" (just as it does not make sense to speak of negative movement, but acceleration and deceleration). Entropy is a quantity that can only grow. Its deceleration gives the impression that it has been reversed, as each accelerating state is able, under certain circumstances, to trigger surprisingly organized processes (for instance, frequent aerobic exercise increases secondary vascularization, producing microvessels that reduce the risk of fatal vascular accidents, although the aging of the organism continues to progress, at best, to natural organ failure).

From there one can already see how much nonsense is expected when searching the subject. Of course, this almost universal appeal to entropy, from thermodynamics to economics, through biology and information theory, reflects at least a feeling that it is fundamental, yet little understood (postmodern sociologists and systemic-minded management theorists use entropy in a way that promotes obscurantism rather than enlightenment). There are two main facts that compose the misunderstandings surrounding entropy: firstly, the more technical, concerns the distancing from its thermodynamical origins; secondly, the more subjective, concerns the excesses of voluntarist and imaginationist idealism. In the first case, issues are discussed by an unqualified quorum. In the second case, speculation comes not from scientific plausibility, but from gratuitous fiction.

Serious and competent authors have defined entropy in slightly different but equivalent ways. Ultimately, we can understand it as 1) the magnitude that quantifies the thermodynamical degradation of a system, and 2) the quantity that describes the incapacity of a system to process (convert) energy. Hence it is seen that remote associations of entropy with degenerating systems must go through thermodynamics, albeit indirectly, but not by mere formal analogies.

It is true that all human activity on the planet consumes energy. The problem is how to formalize this consumption in terms of the second law of thermodynamics, when the theoretical physical aspects of the system in question are not so evident. Depending on the scale of the system, however, it is possible to create a thermal emissivity representation; an urban system would be a good example. A great city, being the center of complex and diversified human relations, bears a thermodynamical image if we think of the heat dissipated in traffic, civil construction works, industrial machines, mobile telephony, human bodies, residential lamps, public lighting, and so on. However, the main objective of this work is to construct not a complete thermodynamical image but a starting point for more precise researches on the control variables necessary for an effective urban management of energy and wastes from its dissipation.

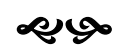




\section{Methods and Applications}

Climatology is a very complex science, so if we were to analyze urban climatic phenomena in their particularities, we would most likely find ourselves in trouble in a tangle of possibilities, some of which considered controversial. Fortunately, present macroscopic approach allows us to observe an object with middling homogeneous areas under the prism of its global thermal emissions, something that considerably simplifies the analysis in progress.

There is no scarcity of imagery devices to aid modern urban climate studies. With the increasing development of satellite imagery systems, the images produced have come to constitute an important tool for observing and analyzing climatic phenomena, particularly with respect to urban environments. Images from the NOAA / AVHRR satellite (spatial resolution of $1.1 \mathrm{~km}$ at Nadir) are applicable to climatic studies of large urban centers, while images from the Landsat 5 and 7 satellites, with spatial resolutions of 120 and 60 meters, respectively, have provided support to the study of configuration and thermal variation in the intra-small size. Faced with so many resources, several authors have been working on the theme of urban heat $[3,4,5,14]$.

The concept of caloric field is in fact a deepening of the idea of thermal field $[10,11,12,13]$ with the main difference that the field is described in its evolution as being a complex scalar associated with its own entropy by a field equation,

$$
\partial_{q} \partial^{q} \xi+1-\gamma^{2} \xi-2 \gamma^{2} \xi \ln |\xi|=0,
$$

where $\xi$ is the field and $\gamma$ is the opacity of the medium. In fact, this construct may be generalized to varied contexts of thermal irradiation, although the gauging only manifests beneath an intense field, where thermochemical transformations occur from a certain amount of matter in interaction with the field ${ }^{2}$. That equation of field evolution is deduced from a Lagrangian density whose role is precisely to establish a coded symmetry for derivation of the equations of "motion" of the system in question. The complex scalar representation was chosen to ensure an always positive entropy according to the field equation; also, it generalizes the formalism so that, when gauging is applicable, it becomes technically useful. So, for instance, let us take the caloric field

$$
\xi=e^{i 2 n \gamma q-\vartheta}
$$

with its conjugate

$$
\xi^{\dagger}=e^{-i 2 n \gamma q-\vartheta},
$$

where $q$ is the generalized coordinate, $n$ is the polytropic index, and $\vartheta$ is the refractive index of the medium. The polytropic regime assumption is in fact a simplifier hypothesis ${ }^{3}$ [13]. Lagrangian density is given by

\footnotetext{
${ }^{2}$ In the theory of caloric fields, the gauge field is closely linked to the appearance of the so-called "minimal thermal mass factor of dynamic interaction", which responds by a massive feedback to the original field due to the thermochemical interaction between field and matter under high temperatures.

3 To the real conditions of the troposphere of Earth corresponds the state with the polytropic thermal stratification. I assume that the whole atmosphere has a polytropic stratification (vertically finite).
} 


$$
\mathcal{L}=\partial_{q} \xi^{\dagger} \partial^{q} \xi-|\xi|^{2}+\gamma^{2}|\xi|^{2} \ln |\xi|^{2} .
$$

Application of Noether's theorem shows that there is a conserved caloric strength $Q$,

$$
\begin{gathered}
\frac{\partial \mathcal{L}}{\partial\left(\partial_{q} \xi\right)^{\dagger}}\left(\partial_{q} \xi\right)^{\dagger}+\frac{\partial \mathcal{L}}{\partial\left(\partial^{q} \xi\right)}\left(\partial^{q} \xi\right)-\mathcal{L}=Q \\
\frac{\partial \mathcal{L}}{\partial \partial_{q} \xi^{\dagger}} \partial_{q} \xi^{\dagger}+\frac{\partial \mathcal{L}}{\partial \partial^{q} \xi} \partial^{q} \xi- \\
-\partial_{q} \xi^{\dagger} \partial^{q} \xi+|\xi|^{2}-\gamma^{2}|\xi|^{2} \ln |\xi|^{2}=Q \\
\partial_{q} \xi^{\dagger} \partial^{q} \xi+\partial^{q} \xi \partial_{q} \xi^{\dagger}-\partial_{q} \xi^{\dagger} \partial^{q} \xi+ \\
+|\xi|^{2}-\gamma^{2}|\xi|^{2} \ln \mid \xi^{2}=Q_{.}
\end{gathered}
$$

Derivatives of both fields are

$$
\begin{gathered}
\partial^{q} \xi=i 2 n \gamma e^{i 2 n \gamma q-\vartheta} \\
\text { and } \\
\partial_{q} \xi^{\dagger}=-i 2 n \gamma e^{-i 2 n \gamma q-\vartheta} .
\end{gathered}
$$

Second derivative of the first field is

$$
\partial_{q} \partial^{q} \xi=-4 n^{2} \gamma^{2} e^{i 2 n \gamma q-\vartheta}
$$

Substituting in field equation, it follows

$$
\begin{aligned}
& -4 n^{2} \gamma^{2} e^{i n \gamma q-\vartheta}+1-\gamma^{2} e^{i 2 n \gamma q-\vartheta}+ \\
& +2 \gamma^{2} \vartheta e^{i 2 n \gamma q-\vartheta}=0
\end{aligned}
$$

$$
\begin{gathered}
-4 n^{2} \gamma^{2}+2 \gamma^{2} \vartheta-\gamma^{2}+1=0 ; \\
\gamma^{2} 2 \vartheta-4 n^{2}-1=-1 ; \\
\gamma=\frac{1}{\sqrt{4 n^{2}+1-2 \vartheta}} .
\end{gathered}
$$

This is the expression of the opacity as a function of polytropic index and refractive index, remembering that the range of interest of the polytropic index is limited to $0 \leq n \leq 5$. For the air, with a significant degree of humidity, we may consider $n=1.2$, which is approximately the value observed in Earth's atmosphere. Assuming the refractive index of the air equal to 1.0003 and substituting both values in expression (3), we obtain

$$
\begin{gathered}
\gamma=\frac{1}{\sqrt{4 \times 1.2^{2}+1-2 \times 1.0003}}= \\
=0.458378
\end{gathered}
$$

This is the mean Earth's atmosphere opacity in normal conditions. It is noteworthy that field entropy, given by

$$
S=\int-2 \gamma^{2}|\xi|^{2} \ln |\xi| d q
$$

is positive for any value of the field. To check this, take the expressions

$$
|\xi|^{2}=\xi \xi^{\dagger}=e^{i 2 n \gamma q-\vartheta} e^{-i 2 n \gamma q-\vartheta}=e^{-2 \vartheta},
$$


and replace in equation (4),

$$
S=\int 2 \gamma^{2} e^{-2 \vartheta} \vartheta d q=0.05685 q
$$

The generalized coordinate is a time function, not the time " $t$ " of clocks but the global evolutional time " $\tau$ " valued in the interval $[0,1]$. Within this very small range, the growth of entropy can be excellently described by

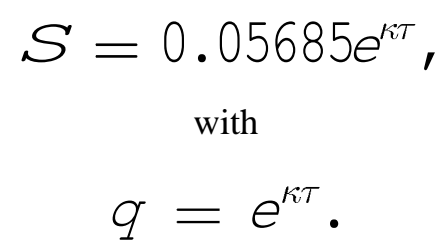

By definition, the rate of entropy variation is given by

$$
\frac{d^{2} S}{d \tau^{2}}=0.05685 \kappa^{2} e^{\kappa \tau}
$$

with $\kappa$ being the average Sky View Factor (SVF) of the city. Since the entire lifetime of a city is undefined, and since the global evolutional time range is very small, we can assume that the variable $\tau$ has "now" a very small value4 in generic time Units (tU), which would allow us to approximate the exponential function to 1. According to the table furnished by Middel et al [5], I adapted another table with the rate of entropy for some cities in the world (Table 1).

\footnotetext{
${ }^{4}$ In fact, it is possible to relate clock time $t$ to $\tau$ via Green's functions, but this is not the case to do this in present paper.
}

\section{Discussion}

Measuring the field we can evaluate the entropic trail that it leaves, and so, comparing different trails among distinct climatologically similar cities, we can establish a parameter of weighting indicative of the volume of irreversible processes that affect the environment in the immediate vicinity of each city. Note that the advancement of entropy was analyzed here only in terms of assumed natural environmental conditions. Both the refractive index and the opacity of the medium may vary significantly for anthropogenic reasons, thus affecting the rate of change of entropy. In this way, caloric field is only understood in its interaction with matter insofar as the only thing observed is the degradation of the system, not the entropy, not even the degradation of the field itself.

In present study, the concept of heat island was implicit in the entire large city. Important contributions in modeling local islands of heat were brought by Oke $[6,7,8,9]$, among which the relation between height-distance of buildings that led to the use of the above referred technique known as the SVF, expressed through the equality

$$
\mathrm{dT}_{\max }=15.27-13.88 \times \mathrm{SVF},
$$

where $\mathrm{dT}_{\max }=$ intensity of the heat island $\left({ }^{\circ} \mathrm{C}\right)[8]$. According to this formula, the author argues that the island of heat is increased or reduced because of the loss or heat gain of the radiation by the "obstruction index" of the sky. The SVF is still widely used today as one of the most efficient urban spatial indicators for radiation and thermal environmental assessment [14]. The obstruction index is similar to the blurring $\gamma$-factor (opacity) of the medium in the former caloric field 
theory. In the original equation of the caloric field, the quantity $1-\gamma^{2}$, named "luminothermic capacity", acts on the field to express the influence of the environment. The opacity may be considered both from the point of view of the radiation that arrives from an external source, and from the radiation returned to the medium from a diffusing source heated on the terrestrial surface.

Lastly, it's interesting to note that conserved caloric strength implies

$$
\frac{d}{d \tau}\left[\partial_{q} \xi^{\dagger} \partial^{q} \xi+|\xi|^{2}-\gamma^{2}|\xi|^{2} \ln |\xi|^{2}\right]=0 .
$$

To verify, let us substitute the derivatives,

$$
\frac{d}{d \tau} 4 n^{2} \gamma^{2} e^{-2 \vartheta}+e^{-2 \vartheta}+\gamma^{2} 2 \vartheta e^{-2 \vartheta}=0
$$

which is true for any global time.

\section{Conclusion}

Present article showed a new approach of urban entropy based on the former concept of caloric field developed by Serpa (2016). From the field equation, considering only thermal effects of solar radiation on the urban environment, it was possible to give a satisfactory relation between the refractive index and the opacity of the medium, from which entropy and its rate of growth were obtained. Both opacity and refractive index largely mirror the level of local pollution. Also, the work discussed a global time variable as the most adequate to treat entropy, since the conventional lifetime of a city is undefined. It is hoped that this approach shall win adepts to improve the model with inclusion of anthropogenic parameters in order to build a more complete representation that helps studies in environmental economics and urban ecology. It is also expected that the model shall be applied to Brazilian cities.

\section{References}

[1] Bunge, M. 1973. Philosophy of Physics, 1. ed. Boston, D. Reidel Publishing Company.

[2] Bunge, M. 2002. Dicionário de Filosofia, 1. ed. São Paulo, Editora Perspectiva.

[3] Li, X.; Li, W.; Middel, A.; Harlan, S.; Brazel, A.; Turner, B. 2016. "Remote Sensing of the Surface Urban Heat Island and Land Architecture in Phoenix, Arizona: Combined Effects of Land Composition and Configuration and Cadastral-Demographic-Economic Factors." Remote Sens. Environ, v. 174, p. 233-243.

[4] Gál, T.; Unger, J. 2014. "A New Software Tool for SVF Calculations Using Building and Tree-Crown Databases." Urban Climate, v. 10, p. 594-606.

[5] Middel, A. et al 2018. "Sky View Factor Footprints for Urban Climate Modeling." Urban Climate, v. 25, p. 120-134.

[6] Oke, T. 1978. Boundary Layer Climates. 2. ed. London, Ethuen \& CO.

[7] Oke, T. 1981. "Canyon Geometry and the Nocturnal Urban Heat Island: Comparison of Scale Model and Field Observations." Journal of Climatology, v.1, p. 237-254.

[8] Oke, T. 1982. "The Energetic Basis of the Urban Heat Island." Quat. J.R. Met. Soc., v.108, n455, p. 1-24.

[9] Oke, T. 1988. "Street Design and Urban Canopy Layer Climate." Energy and Buildings, v.11, p. 103-113. [10] Serpa, N.; Costa, I.; Franco Gonçalves, R. 2016. “A Thermal System Based on Controlled Entropy for Treatment of Medical Waste by Solar Energy." IFIP Advances in Information and Communication Technology, v. 488, p. 789-797.

[11] Serpa, N. 2017. "Controlled Entropy and Gauge Theory in Recycling Condensed Matter by Solar Energy." Journal of Physical Science and Appication, v. 7, p. 6-14.

[12] Serpa, N. 2017. "Thesaurus Theoriis Circa Gravitatis et Cetera." CALIBRE - Revista Brasiliense de Engenharia e Física Aplicada, v. 2 supl., p. 3-78. 
[13] Serpa, N. 2018. "Sur l'Entropie Contrôlée des Systèmes: Transformations de la Matière Condensée (On the Controlled Entropy of the Systems: Condensed Matter Transformations)." CALIBRE - Revista Brasiliense de Engenharia e Física Aplicada, v. 3 supl., p. 1-123.

[14] Zeng, L.; Lu, J.; Li, W.; Li, Y. 2018. “A Fast Approach for Large-Scale Sky View Factor Estimation Using Street View Images.” Building and Environment, v.135, p. 74-84. 
Table 1 - Average entropy rate for some cities.

\begin{tabular}{|l|r|r|}
\hline \multicolumn{1}{|c|}{ CITY } & SVF (average) & AVERAGE ENTROPY RATE $\left(\mathbf{J} /{ }^{\circ}\right.$ K.tU $\left.{ }^{2}\right)$ \\
\hline Manhattan & 0.545 & 0.016886 \\
\hline Paris & 0.586 & 0.019522 \\
\hline Singapore & 0.595 & 0.020126 \\
\hline Seoul & 0.680 & 0.026287 \\
\hline Tokyo & 0.693 & 0.027302 \\
\hline Vancouver & 0.713 & 0.028901 \\
\hline Philadelphia & 0.720 & 0.029471 \\
\hline Bonn & 0.746 & 0.031638 \\
\hline San Francisco & 0.811 & 0.037391 \\
\hline
\end{tabular}

Source: adapted by the author from Middel et al, 2018. 